Volume 9, No.4, July - August 2020

International Journal of Advanced Trends in Computer Science and Engineering

Available Online at http://www.warse.org/IJATCSE/static/pdf/file/ijatcse03942020.pdf

httns://doi.org/10.30534/iiatcse/2020/03942020

\title{
Design and Development of Wireless Fan Regulator using ZigBee Concept
}

\author{
Anita Gehlot ${ }^{1}$, Rajesh Singh ${ }^{2}$, Amit Kumar Thakur ${ }^{3}$, Rajeev Pandey ${ }^{3}$ \\ ${ }^{1}$ Lovely Professional University, India, eranita5@gmail.com \\ ${ }^{2}$ Lovely Professional University, India, srajssssece@ gmail.com \\ ${ }^{3}$ Lovely Professional University, India, amitthakur3177@gmail.com \\ ${ }^{4}$ Lovely Professional University, India, rajeevpandeyrs@ gmail.com
}

\begin{abstract}
Due to rapid advances in wireless communication, wireless sensor networks, and information technologies; it is now possible to integrate a level of smartness in the home environment. This paper deals with the proposed design and its implementation required to Wireless Fan Regulator. The main use of this regulator is to adjust the rotation speed of the fan using remote consisting of Zigbee, potentiometer, microcontroller, LCD at the transmitting section (remote unit), and Zigbee, LCD and dimmer at the receiving section (Slave unit). The device would allow a person to control the rotating speed of a fan by simply pointing the remote potentiometer or by pressing any key on the remote. The key target is that it can easily work approximately at a distance of 60 meters from the fan. There are two endpoints used, one is to transmit and the other one is to receive. At the transmitting section Zigbee module is used to transmit the data and at the receiving section also Zigbee module is used to receive the data and it will also display the same on LCD. The requirements of the power consumption are less in Zigbee communication i.e this communication is used in this paper. Since the transceivers power consumption is very less in Zigbee protocol communication so it's pretty obvious that Zigbee Protocol communication is the prime choice for smart object development.
\end{abstract}

Key words: Sensor nodes, Zigbee, dimmer, microcontroller.

\section{INTRODUCTION}

Home automation is a technology that helps users with a voice to control household electrical devices, whether they are elderly or disabled [1]. Wireless network sensor routing (WSN) plays a vital role in implementing node mobility. While implementing WSN's mobility principle extends the range of applications, network connectivity is greatly affected. In the mobile WSN, the topology of the network changes [2]. With tremendous growth in Wi-Fi, 5 G cellular and Wireless-sensing, wireless networks have become popular in recent years. The common problem we found in the aforementioned technologies is scalability and survivability would affect device performance. The current evolution requires an improvement in traditional topologies [3].

There are many drawbacks to the current developed event detection and tracking systems that make them unsuitable or effective for use in smart homes. Such weaknesses include addressing change of direction and varying speeds of an unfolding event, tolerance of node faults, energy usage, lack of target, precision monitoring, target dynamics, and accuracy prediction [4].Wireless Sensor Networks (WSNs) emerge as a rich area of active research, involving various aspects. Nowadays, sensors are used as applications which can track and control in many domains. These domains include applications like climate, home, industry, military, safety, etc. WSNs are used in numerous applications which require safe and confidential use of sensitive information. This paper provides comprehensive details regarding WSN constraints, vulnerabilities, and attacks on security. Additionally, the paper discusses the recent countermeasures used in terms of their achievements and weaknesses to counter WSN attacks [5].

This paper uses a tree-shaped lightweight protocol to control the energy consumption by sensor nodes in Wireless Sensor Nodes (WSNs). The key goal is to increase the network's life span by raising the energy usage. The proposed network routing protocol is used to address the routing problems associated with mobile stations in infrastructural networks [6].Usually, wireless sensor networks consist of a large number of small low-cost electronic devices with limited ability to collect information from a specified interest range. Thus, a network's lifespan is shortened, and energy usage is increased. This paper mainly aims to activate the node and reduce energy consumption to send or receive information from the nodes of the sensors. The paper considers three variables to pick as a cluster head based on a genetic algorithm, such as residual energy, distance from the sink node, node density [7].

In this study, a water level regulation system based on the Wireless Sensor Network (WSN) architecture is proposed. WSN architecture consists of three nodes, such as sensor node, cluster head node, sink node, etc. The sensor node 
deployed in the paddy field senses the water level data and communicates to the sink node with the help of the float sensor and the ZigBee wireless communication module [8]. A wireless technology is used in this setup with the support of the ZigBee and AvR microcontrollers. This system enables a person sitting at any place to join his or her family doctor or the nearest hospital on an ongoing basis by means of diagnostic information. Should heart defects occur, the person should alert the nearest doctor with whom we link our WSN system to ensure that we receive the response at the earliest opportunity [9]. The ZigBee module for the establishment of wireless communication between transmitter and receivers is used in the proposed framework. In the Nuclear Power Plant design and evaluation the proposed advanced control room is being considered [10].

These days, there is no building without electricity. Each house and industry consumes a great deal of electricity, and it is a huge trouble for a record keeper to maintain this energy log. A concept for the user logging of this unit data is proposed in this project. The energy meters currently used are digital, so a computer system for monitoring and recording data is provided remotely. This machine uses the communication protocol ZigBee and the RF IC-CC2500, 2.4GHz(ISM) transceiver. [11] Low power and low cost deployment make the Wireless Sensor Area Network a dominant alternative and cloud-based field monitoring system. A method has been developed, for example, for agricultural systems, which functions as phonological indicators of the various seasonal field developments and the number for wide area areas of IoT (the Internet of Things) of regional growth environment effects [12]. In order to build smart homes, the wireless sensor networks (WSNs) have become indispensable. This paper is aimed at creating such a WSN that smart home systems can be installed. The aim is to develop and implement the Wireless Sensor Node and the ZigBee Technology-based coordinator [13].

In the next few years, the proportion of the elderly in every population is increasing rapidly, and this trend will not change [14].This paper addresses the nature, production and application of Zigbee-based temperature sensors. The key purpose of this paper's study is to feel the temperature and to demonstrate the effects using Zigbee technology on the LCD [15].The two main technologies that have developed innovative research and engineering techniques are Radio Frequency Identification (RFID) systems and Wireless Personal Area Network (WPAN).These technologies are different and have different applications, but if they are integrated, several doors will be opened in research [16].

\section{WIRELESS SENSOR NETWORK}

This section is all about the introduction of the work and it will also describe the Wireless Sensor Network (WSN), Zigbee, potentiometer, and dimmer.Figure1 illustrates the block diagram of the complete system.

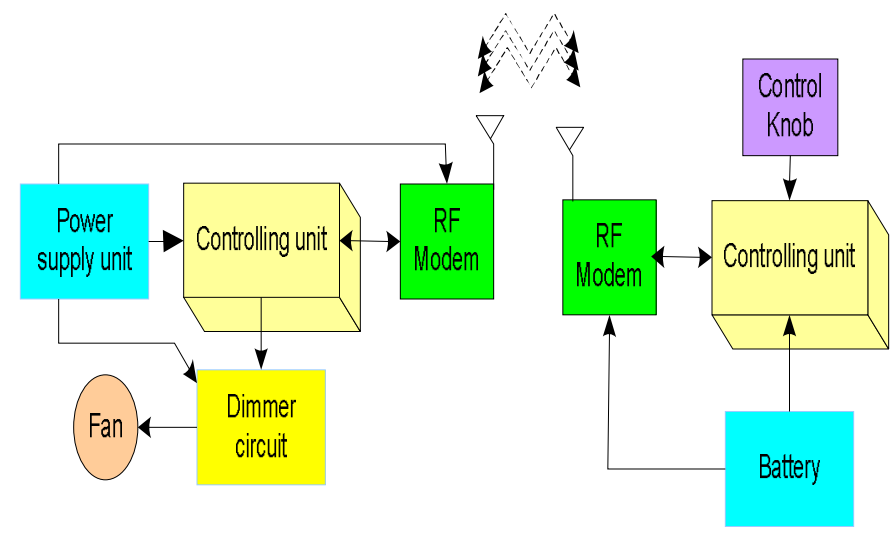

Figure 1:. Block diagram of the system

A network created by small autonomous sensors (nodes) is known as a wireless sensor network (WSN). A radiofrequency system, i.e. used by the nodes to transmit the information collected through the sensors to the central processing unit (CPU).The communication between a node and the CPU might happen either directly or step by step through different nodes within the network.

There are five key parts of a sensor:-

- The data collected from the environment is done through one or more sensors.

- The task management is done by the central unity as a microprocessor.

- Communication will be established by the use of a transceiver.

- Temporary data created at the time of processing will store into memory.

- The power or energy provided to all parts through the battery.

\subsection{Sensor network in smart homes}

Since technology is growing day by day. So no one wants to do regular work with the manual appliance. So to overcome these manual works, automation takes place. In automation, Sensor network plays a vital role. The sensor network is used to make appliances smart such as smart vacuum cleaner, smart microwave ovens, smart refrigerators, and smart television, etc. There are several different nodes inside the home appliances and they can communicate with each other internally, and through the Internet or satellite, it can interact with the external network. By all this, an end-user can easily operate their device locally and remotely.

\section{ZIGBEE}

It's a network protocol in which work can work in the wireless medium. It is specially manufactured controllers and sensors that have a low data rate. It's a combination of the hardware, software, and the different services and is designed to work as a common networking system that follows wireless 


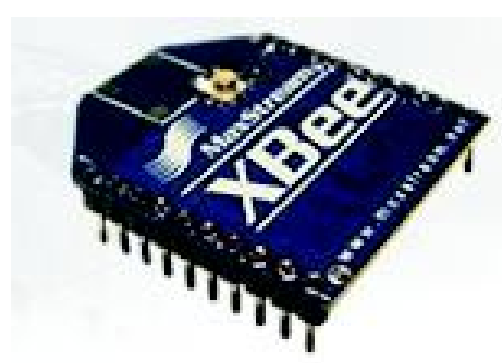

Figure 2:Zigbee

protocol for different Sensors and different controllers. Though there much other wireless protocol but they deal with a huge amount of data. Due to this reason, Zigbee is appropriate for the machine or device which is of prime importance. Fig 2 shows the Zigbee. The less cost, more reliable, more secure, less power consumption, less complex, and interpretability with different devices of Zigbee, these all make more selective networking protocol for any device. It also provides three bands of frequency for any operation, since it works on the low power consumption. The other driving factors are low cost, high reliability, high security, low battery usage, simplicity, and 2.8 volt-3.3volt is sufficient range to work.

Zigbee product is also beneficial from the maintenance point because Zigbee product has a key feature that if any kind of malfunctioning will take place then it will provide an alert from the product. Zigbee has many ways of use to control any system or appliance such as an emergency switch, alert system, automatic light system, etc.

\subsection{Working of Zigbee}

A microcontroller of eight-bit along with a transceiver with fewer amounts of flash memory and RAM is implemented on the Zigbee. It provides its operations on the frequency at ISM $2.4 \mathrm{GHz}$. Its pins are also suited for the product of max stream Zigbee. For the communication purpose, ZigBee uses three radio frequencies according to the coverage of areas respectively. For international or world-wide coverage it works with the $250 \mathrm{kbps}$ data speed through 16 channels at the frequency of 2.4GHz.For Europe, it provides a data speed of $20 \mathrm{kbps}$ through a single channel at the frequency of $868 \mathrm{MHz}$. And for America, it provides the data speed of 40kbps through 10 channels at the frequency of $915 \mathrm{MHz}$. There are three radio frequencies used for Zigbee radio frequency communications $2.4 \mathrm{GHz}$ with 16 channels and a data rate of $250 \mathrm{kbps}$ for worldwide coverage, $868 \mathrm{MHz}$ with a single channel and a data rate of $20 \mathrm{kbps}$ in Europe and $915 \mathrm{MHz}$ with 10 channels and a data rate of $40 \mathrm{kbps}$ in America. If we compare the data speed of the Zigbee with Bluetooth at $250 \mathrm{kbps}$, it is one-tenth. $64 \mathrm{k}$ around 65,536 network nodes can hold at the Broadcasting range of 70 meters nearly for Zigbee theoretically. Not tests in the world reached this level yet. Since Zigbee follow a zigzag path for the communication.

\subsection{Uses of Zigbee}

There are a huge amount of uses and advantages and of Zigbee protocol especially in creating an automatic network system, System related to home security, industrial networking control system, Pc connectivity with the surrounding devices, monitoring of far distance devices, and many other.

- Security system and lighting control.

- Automaticity of houses and control systems in the building.

- House appliances and an alarm system in of fire.

- Monitor long-distance devices or systems.

- In embedded system network capturing data from the sensor.

\section{POTENTIOMETER}

A voltage variable is also known as a potentiometer. It looks like a three-terminal pot. So that's why it also is known as a three-terminal resistor. It has a variable voltage divider through a sliding contact. In case if there is the only use of two-terminal then the third terminal is used as a variable resistor or a rheostat.

There are different uses of the potentiometer, but basically, it is used to compute the electric potential (voltage). So the components which are working this principle are use potentiometer. An electric device, there are widely used such as in audio device to adjust the volume. In some other devices, it is used for different purposes. For example, in the joystick, it is used as a position transducer. On the point of power dissipation potentiometer is much corresponding to the controlled load. So this the reason that it is hardly used forthwith control noteworthy power (greater than a watt).

\section{DIMMER}

A device that is used to adjust the light's brightness is known as Dimmers. To change the output intensity of the light, mean power should be varied and that can be achieved by changing in RMS voltage, either decrease or increase. Since there are different uses of the voltage variable devices. But dimmer is basically to control output intensity from LEDs(light-emitting diodes), CFLs(Compact fluorescent lights), and resistive incandescent halogen. For varying the intensity of fluorescent, solid state, mercury, and else arc lighting, special kind equipment required.Its range varies according to the requirement, from small unite of size(House purpose) or low power to a large unit of size( Theater lighting installations) high power. House purpose dimmer can be controlled through the remote system.

DMX or DALI is a digital control system. In advance, fine dimmers could be controlled through these control systems.Now, these protocols used with the combination of 
ethernet. In the advanced industry of lights, "fades" are knowns variations in the intensity of light. Either it will "fade up" or "fade down". With the manual, there is a problem that the speed of varying the speed of light is very slow. But by the use advance digital system, this problem gets eliminate( But maybe the life of the lamp gets reduce). At the place of variable resistor, SCR(silicon-controlled rectifiers) is used to manufacture the advanced modern dimmers. Higher efficiency is the reason behind it. It also that power loss is more in the variable resistor in the form of heat. Since SCR works on two states only one is "on" state and the other one is "off" state, this based on low and high resistance respectively

\section{HARDWARE DESCRIPTION}

Block diagrams of the proposed system are given below:

\section{Transmitting Node section:}

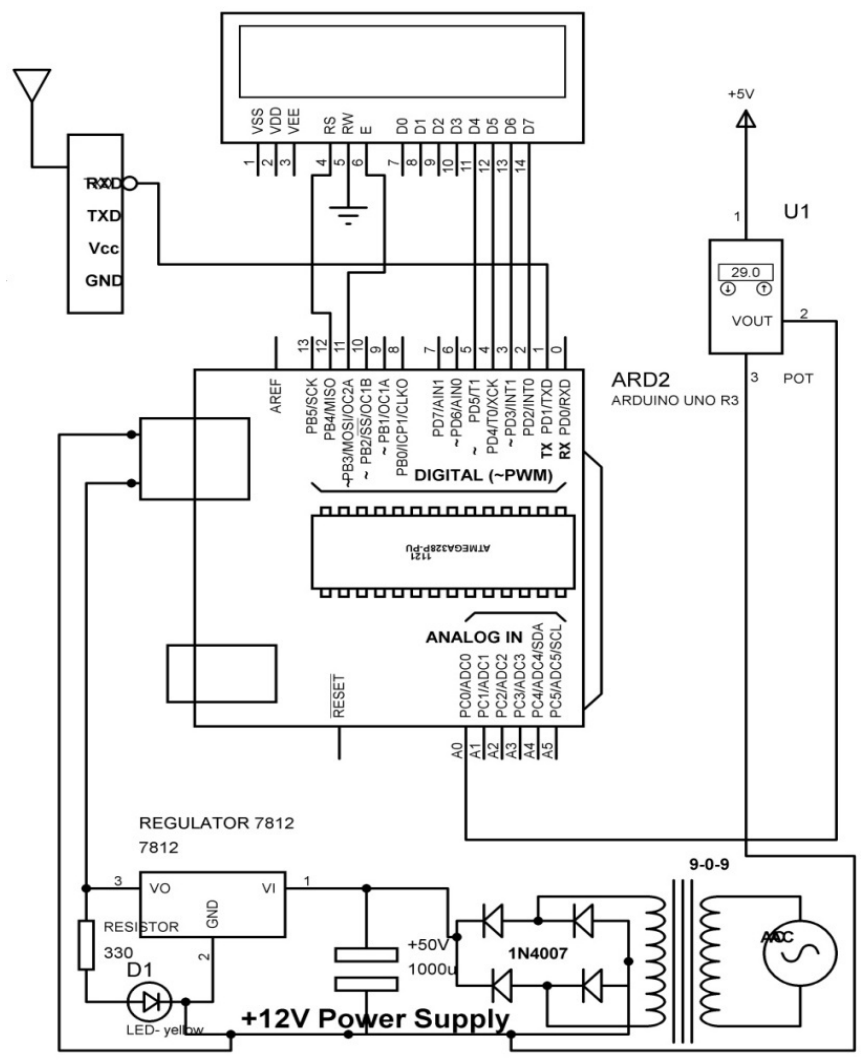

Figure 3: Block Diagram of the transmitter section

Figure 3 shows the block diagram of the transmitter section. A POT is connected to the ADC pins of the microcontroller. POT is used to vary the speed of the fan or to regulate the fan to low or high speed. The ADC converts this analog data to digital form and the speed of the fan is adjusted to some levels of ADC, for eg. At ADC level 120 fan regulator will be at position 1, for level 240 position is 2 , for level 360 position is 3 , for level 550 position is 4 , for level 720 position is 5 . The data or the adjusted position will be displayed at the LCD. Whip antenna help to transmit the data from Zigbee. The power supply of $2.8 \mathrm{~V}$ to $3.3 \mathrm{~V}$ is designed for the operation of Zigbee is manufactured to work at a voltage of range $2.8 \mathrm{~V}-3.3 \mathrm{~V}$. The Zigbee at the receiving side receives the transmitted data. The transmitting node is connected to the remote which is held by a person at a distance and by pressing a button on that remote, speed is adjusted. Similarly, more than one potentiometers can be connected to the microcontroller to adjust the speed of other fans.

\section{Receiving node section:}

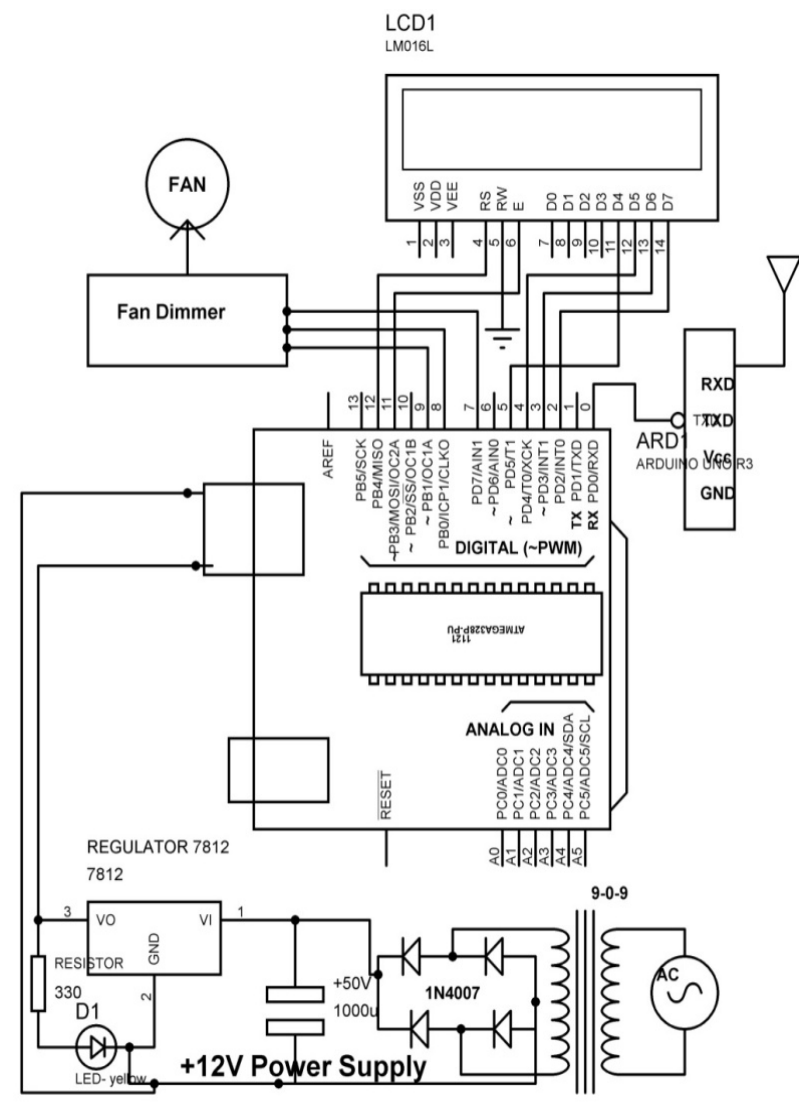

Figure 4: Block diagram of receiving node

A similar block diagram as in Figure 4 is designed for receiving Zigbee as in the transmitter as shown in fig. This data is fed to the ATMEGA microcontroller through the Rx pin. The serial data received is converted into parallel data by the inbuilt USART and is displayed on the four-byte LCD. The dimmer is connected to the ATMEGA microcontroller. When the speed goes high or low, dimmer is activated and the speed of the fan is adjusted through the dimmer circuit and the data will be displayed on the LCD.

The receiving node will be attached to the fan and the fan speed will be wirelessly monitored through the Zigbee module.

\subsection{Components in Node design}

Microcontroller: For the sensor nodes, mostly microcontrollers are used. ATMEGA16 is a controller of the ATMEL family. Since its design is based on CMOS with reduced instruction set computer (RISC) architecture. So its 
power consumption is very low. It has a flash memory of $16 \mathrm{kbytes}$ and one-timer of 16 bit and two-timer of $8 \mathrm{bit}$. There are $32 \mathrm{GP}$ (general purpose) pins are available working. It has 32 general-purpose working registers. The main purpose is of the microcontroller to connect or make a conjunction to make a complete unit.

Transceivers: For a sensor node transmitter and receiver both are essentially required.

- To combine both the task practically and formed a single entity, Transceivers come into the role.

- Since transmit and receive the signal never be possible for wireless medium. So getting out of this problem, a half-duplex operation is a better way.

- Transceiver States should Transmit, Receive, Idle (Ready to receive, but currently not receiving), Sleep (Noteworthy parts of the receiver are switched off).

Power supply: It should be power-efficient: It means to use less amount of resource to get more power)
Alternatives:
- Primary Batteries: it should be not rechargeable
- Secondary Batteries: it must be rechargeable Requisite:
- Potential: It should be high at small weight, small volume, low price
- Potential under load (withstand various usage patterns)
- Self-reprieve or discharge (only in case of low)
- Systematized recharging
- Unwinding (proper use of 'self-recharging effect')
- Voltage Steadiness (convert DC to DC)

\section{SOFTWARE DESCRIPTION}

In making any product advance, software plays a vital role. In this project " $\mathrm{C}$ " language is used to write the program to work the system. For the compiling purpose, AVR studio4 is used. Because AVR studio4 is firmware which is freely available for window and Linux Operating System (OS). Since AVR studio4 is capable to manage all kinds of AVR functions such as ADC, timer, UART, interrupt etc. so that it provides a space to write in embedded ' $C$ ' programming language. Once programmed get finished, then there is a need to generate a hex code file. This hex code file contained the result of the program. And by the use of USB programmer this hex code file gets burnt into the flash memory of the microcontroller. To initialize the microcontroller there is a need to generate an external clock of $14.7456 \mathrm{MHz}$ : There are two fuse bytes are required---- the fuse bytes are as follows:

- C9: It is low fuse byte

- EF: It is High fuse byte

To get the proper functionality software should be written in parts for each subsystem. There are several steps are entangled to program the microcontroller as in Figure 5 to get the required result of wireless personal area network application. So these steps are the following:

- Coding/debugging

- Compiling

- Burning, and

Evaluation

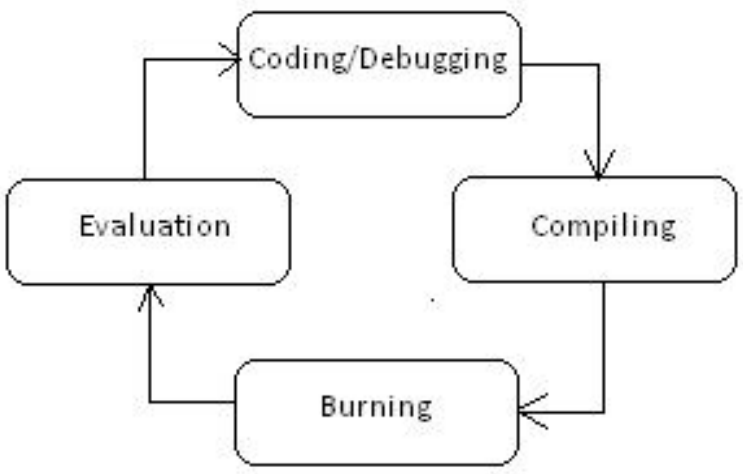

Figure 5: Flowchart for programming the microcontroller

\section{RESULTS AND DISCUSSION}

This proposal suggested the Wireless Fan Regulator and its implementation. This control unit mainly uses Zigbee, Potentiometer, Microcontroller, LCD (remote unit), Zigbee, LCD and dimmer (Slave unit) to adjust the fan's rotational speed using remote control. The system allowed a individual to regulate the spinning speed of a fan by pointing the remote pot or pressing a remote key. The main aim is to be able to operate about 60 meters away from the fan. The Zigbee module transmitted enough data and Zigbee module received data in the transmittal section and also displayed it on LCD on the receiving section. Electricity specifications in the Zigbee contact were found to be smaller. As the usage of power in transceivers in Zigbee protocol communication is quite small, it is very clear that contact with Zigbee Protocol is the first option to build intelligent artifacts.

\section{CONCLUSION}

In this paper, the design of a wireless fan regulator is proposed which can be used to adjust the speed of one or more than one fan from approximately 10 meters away through Zigbee communication. There are 5 levels are provided for speed control of the fan. The main motive of this product to provide ease to the old age people who are not walking. So that they can adjust the speed of fan from their place only. It's also quite beneficial for those who do want disturbance in their sleep. So by using this product they can easily control the speed of their bed only. 


\section{REFERENCES}

[1] Akour M, Radaideh KA, Shadaideh A, Okour O, "Mobile Voice Recognition Based for Smart Home Automation Control", International Journal of Advanced Trends in Computer Science and Engineering, Volume 9, No.3, May - June 2020. https://doi.org/10.30534/ijatcse/2020/196932020

[2] Outazgui S, Fakhri Y, "RE-AODV an Enhancement of AODV Routing Protocol for Wireless Sensor Networks", International Journal of Advanced Trends in Computer Science and Engineering, Volume 8, No.6, November-December,2019.

https://doi.org/10.30534/ijatcse/2019/138862019

[3] Amiripalli SS, Bobba V, "Trimet Graph Optimization, (TGO) based methodology for Scalability and Survivability in Wireless Networks", International Journal of Advanced Trends in Computer Science and Engineering, Volume8, No.6, November-December, 2019.

https://doi.org/10.30534/ijatcse/2019/121862019

[4] Jadaa KJ, Kamarudin LM, Ahmad R., Hussein WN, "Multi Objects Detection and Tracking System for Smart Home using Wireless Sensor Network", International Journal of Advanced Trends in Computer Science and Engineering, Volume 8, No.5, September October 2019.

https://doi.org/10.30534/ijatcse/2019/86852019

[5] Elsadig MA, Altigani A, Baraka MA, "Security Issues and Challenges on Wireless Sensor Networks", International Journal of Advanced Trends in Computer Science and Engineering, Volume 8, No.4, July - August 2019.

https://doi.org/10.30534/ijatcse/2019/78842019

[6] Manikandan V, Sivaram M, "Energy Efficient Dynamic Routing in Wireless Sensor Networks", International Journal of Advanced Trends in Computer Science and Engineering, Volume 8, No.5, September October 2019.

https://doi.org/10.30534/ijatcse/2019/51852019

[7] Abu Salem AO., "A Novel Leach Routing Protocol using Genetic Algorithm for Wireless Sensor Networks", International Journal of Advanced Trends in Computer Science and Engineering, Volume 8, No.5, September - October 2019.

https://doi.org/10.30534/ijatcse/2019/37852019

[8] Singh R, Gehlot A, Thakur AK, Swain M, Akram SV, "Wireless Sensor Network with Power Management System for Water Level Regulation in Paddy Fields" International Journal of Innovative Technology and Exploring Engineering (IJITEE), ISSN: 2278-3075, Volume-9 Issue-7, May 2020

https://doi.org/10.35940/ijitee.G5854.059720

[9] Singh R, Pandey P, Sharma V, "ECG Monitoring System in Wireless Personal Area Network simulation and design using Zigbee Transceiver
Module for Health Care Solution." International Journal of Computer Applications 975: 8887.

[10] Singh R, Sharma M, Kaundal V, "Enhanced Wireless Personal Area Network based Real Time motion (human) data Collection for advanced control room in Power Plants using ZigBee Transceiver Module." International Journal of Emerging Technology and Advanced Engineering, ISSN 2250-2459, Volume 2, Issue 5, May 2012.

[11] Singh R, Sobti S, Bhandula N, Agarwal S, Poonia L, "Wireless Micro Power Meter System up to $100 \mathrm{~W}$ Load Simulation and Design using $2.4 \mathrm{GHz}$ Transceiver Module." International Journal of Engineering Research and Applications (IJERA), ISSN: 2248-9622 www.ijera.com Vol. 2, Issue 4, July-August 2012, pp.051-055

[12] Samkaria R, Singh R, Gehlot A, Choudhary S, "Cloud based agricultural field parameters monitoring using ESP8266 and ZigBee." International Journal of Engineering \& Technology, Vol.7, no. 4-5 (2018): 556-559. https://doi.org/10.14419/ijet.v7i4.5.21156

[13]Xu, M., Ma, L., Xia, F., Yuan, T., Qian, J., Shao, M, "Design and implementation of a wireless sensor network for smart homes" in 2010 7th International Conference on Ubiquitous Intelligence \& Computing and 7th International Conference on Autonomic \& Trusted Computing , pp. 239-243, IEEE, 2010, October.

[14] Gaddam, A. "Wireless sensor network based smart home for elder care". A thesis presented in partial fulfilment of the requirement for the degree of Doctor of Philosophy in Electronics Engineering at School of Engineering and Advanced Technology, Massey University, Manawatu Campus, New Zealand (Doctoral dissertation, Massey University), 2011.

[15] Manjunath, T. C., Kusagur, A., Sanjay, S., Sindushree, S., Ardil, C, "Design, Development \& Implementation of a Temperature Sensor using Zigbee Concepts", World Academy of Science, Engineering and Technology, 44, 2008.

[16] Kaundal, V., Singh, R., Wadhwa, A., Mishra, S., Garg, $\mathrm{T}$, "Wireless personal area network node design with RFID using ZIGBEE transceiver module”, International Journal of Advanced Research in Computer Science and Electronics Engineering (IJARCSEE), 2(2), 193-199, 2013. https://doi.org/10.5923/j.ijee.20120206.02 